\title{
Selective Functional Movement Assessment(SFMA)-based Therapeutic Corrective Exercises Reduces Knee Joint Pain in a Patient with Patellofemoral Pain Syndrome after Pregnancy (Case study)
}

\author{
Jae-eun $\mathrm{Kim}^{1}$ and Jongeun Yim ${ }^{1 *}$ \\ ${ }^{1}$ Department of Physical Therapy, The Graduate School of \\ Sahmyook University, Seoul, Korea \\ jeyim@syu.ac.kr
}

\begin{abstract}
The aim of this study was to determine the effect of selective functional movement assessment (SFMA)-based therapeutic corrective exercise on knee joint pain in a patient with patellofemoral pain syndrome (PFPS) after pregnancy. PFPS is one of the most common causes of knee pain. The present study was conducted in a 35-year-old woman with chronic PFPS following pregnancy. She was seen every other day, 3 times a week for 8 weeks. Physical therapy was performed for tight muscles and fascia in the front, rear, left, and right following mat relaxation, with use of a form roller and corrective exercises suitable for SFMA after simple manual therapy. Corrective exercises were performed with pattern assist, unload, pattern assist with load, and load, based on a $4 \times 4$ matrix and the patient's status and exercise capacity. For each corrective exercise, photographic materials were also provided so that the patient could carry out the corrective exercises at home. The treatment time per session was 50 minutes. Multi-Segmental Flexion showed improvement from Dysfunctional Non-painful (DN) to Functional Non-painful (FN), Multi-Segmental Extension showed improvement from DP to FN, Single Leg Stance (both right and left) showed improvement from DN to FN, and Overhead Deep Squat showed improvement from DP to FN. The Straight Leg Raising (SLR) test showed an increase on the right from $82^{\circ}$ to $90^{\circ}$ and from $63^{\circ}$ to $81^{\circ}$ on the left. The Kujala questionnaire score increased from 63 to 94. The visual analogue scale (VAS) score decreased from 8 to 3. SFMA-based therapeutic corrective exercise was effective for chronic PFPS after pregnancy in this patient
\end{abstract}

Keywords: SFMA, corrective exercise, patellofemoral pain syndrome, pregnancy

\section{Introduction}

Patellofemoral pain syndrome (PPS) is one of the most common causes of knee pain $[1,2]$. A reason may be that the the movement of the patella is unstable during flexion and extension [3]. Studies have also reported that overuse injury, abnormal muscular function, hypermobility of the patella, lateral tightness, and poor flexibility of the quadriceps are also causes [4]. One study compared differences in groups with and without PPS by measuring hip external rotation strength, hip abductor muscle strength, length of the tensor fascia lata myocutaneous flap, length of the quadriceps, hamstring length, gastrocnemius length, and flounder muscle length. The study reported that the group with PPS showed greater deterioration in flexibility according to the difference in the length of quadriceps, hamstring, and gastrocnemius muscle flounder [5]. Thus, the definition of PPS is very broad and imprecise, as indicated by its description as a"syndrome." Many studies have suggested that treatment requires attention to the length of the hamstring. 
The group with PPS showed 145.6 in the length of the hamstring in the supervene position, whereas the control group exhibited 153.7, showing a significant difference [6]. The straight leg raising test was positive at $75.28^{\circ}$ in the group with PPS, and $80^{\circ}$ in the group without PPS [7], which is consistent with the $80^{\circ}$ [8] reported for normal range in functional movement assessment, and the $80^{\circ}$ [9] reported for normal range for the passive straight leg raising test in selective functional movement assessment. A study of the correlation between straight leg raising and PPS reported that hamstring shortening results in quadriceps weakening, eventually causing muscle imbalance and PPS. Another study [10] found that straight leg raising with the stroke ankle in dorsiflexion, while externally rotating the hip in patients with and without PPS, activated the vastus medialis oblique and the vastus lateralis, affecting knee stability. In assessing PPS, a recent study on the dynamic $\mathrm{Q}$ test reported that a dynamic straight leg raising test is more significant than a static test such as the Q angle and navicular drop test [7]. The author also stated that the reliability issue of PPS of the static Q-angle patellofemoral pain syndrome is not structural but instead functional or caused by dynamic imbalance [11-15], 2011; Wolf Petersen et. al). It was reported that the most significant contributor to dynamic imbalance is pain, and that pain inhibits muscle contraction and muscle function [16-18]. Another study reported that pain affects the attitude control strategy, as well as movement [19-21]. Another study found that even after the pain has resolved, bad posture persists [22]. Therefore, the elimination of dynamic imbalance is very important not only for therapeutic implications but also for solving the second problem. Pregnant women report various pains before and after delivery. In particular, PPS occurs because of postural change, joint pressure, and weight change [23]. After pregnancy, many physical and emotional changes occur. Pregnancy protects the fetus through adaptation to physical changes and other factors related to hormonal changes. This causes a change in skeletal muscle, leading to pain [24]. One study reported that pregnancy weight directly affects the knee [25]. Previous studies reported that women within each of the different periods after birth complain of pain and discomfort $[26,27]$. Sources of pain related to childbirth are genital, back, and pelvic. However, detailed studies on these sources of pain are lacking. Therefore, the present case study aimed to evaluate intervention related to pain and movement assessment in a patient with PPS.

\section{Methods}

\subsection{Participant}

The present study was conducted in a 35-year-old woman with chronic IPC after a delivery, who was admitted to B Hospital located in Do-bong-gu, Seoul. The patient reported that she visited the hospital in order to prevent any more injuries in her second pregnancy, as related to her current pain. She visited the hospital every other day, 3 times a week for 8 weeks.

\subsection{Intervention}

Physical therapy was performed for tight muscles and fascia in the front, rear, left, and right following mat relaxation, with use of a form roller and corrective exercises suitable for selective functional movement assessment (SFMA) after simple manual therapy. Corrective exercises were performed with pattern assist, unload, pattern assist with load, and load, based on a $4 \times 4$ matrix and the patient's status and exercise capacity. [28]. For each corrective exercise, photographic materials were also provided so that the patient could carry out the corrective exercises at home (Table 1 ). The treatment time per session was $50 \mathrm{~min}$. If pain was present in the knee or the treatment seemed excessive, the patent was allowed to use an ice pack. 
Table 1. Corrective Exercise

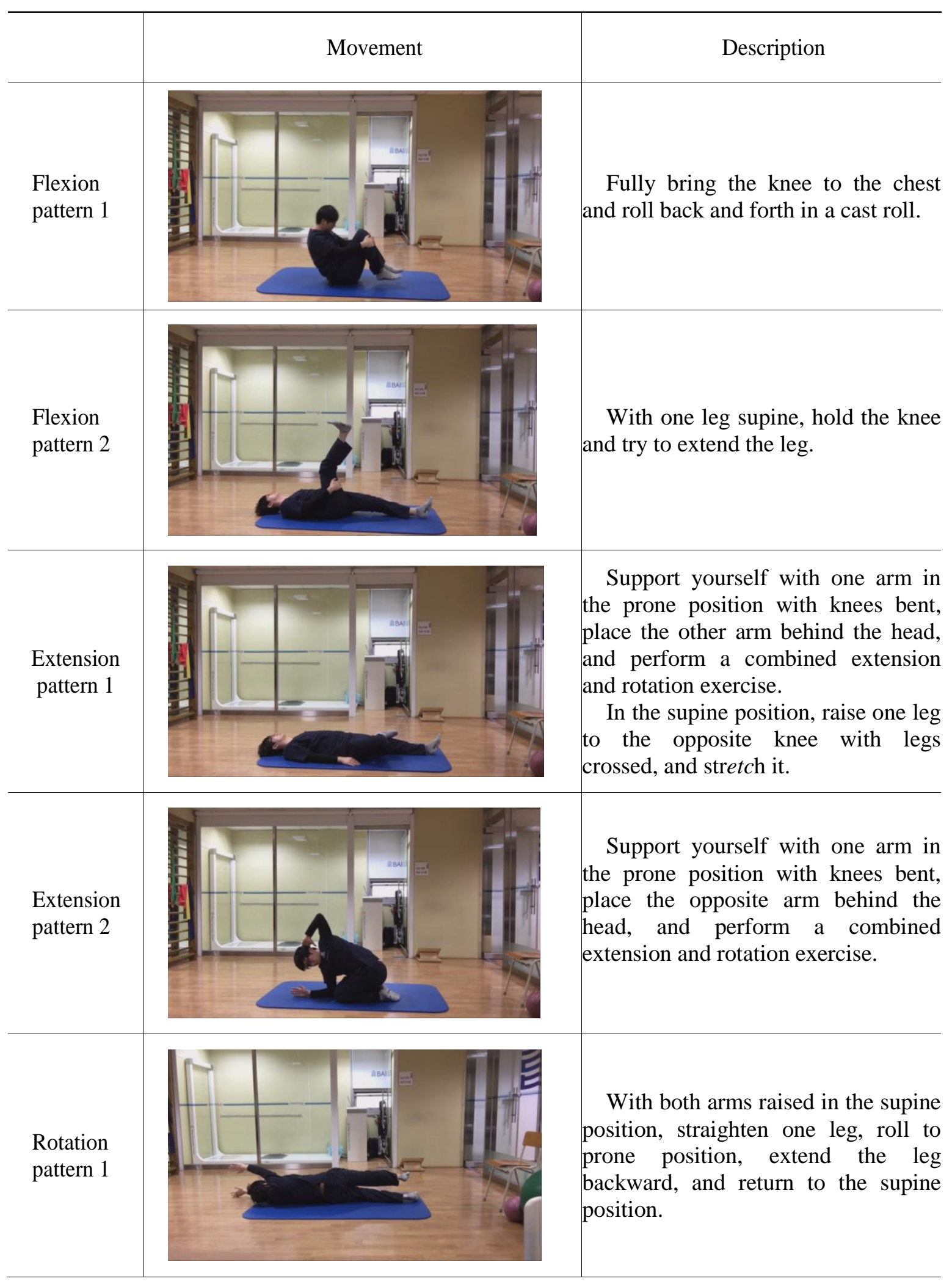




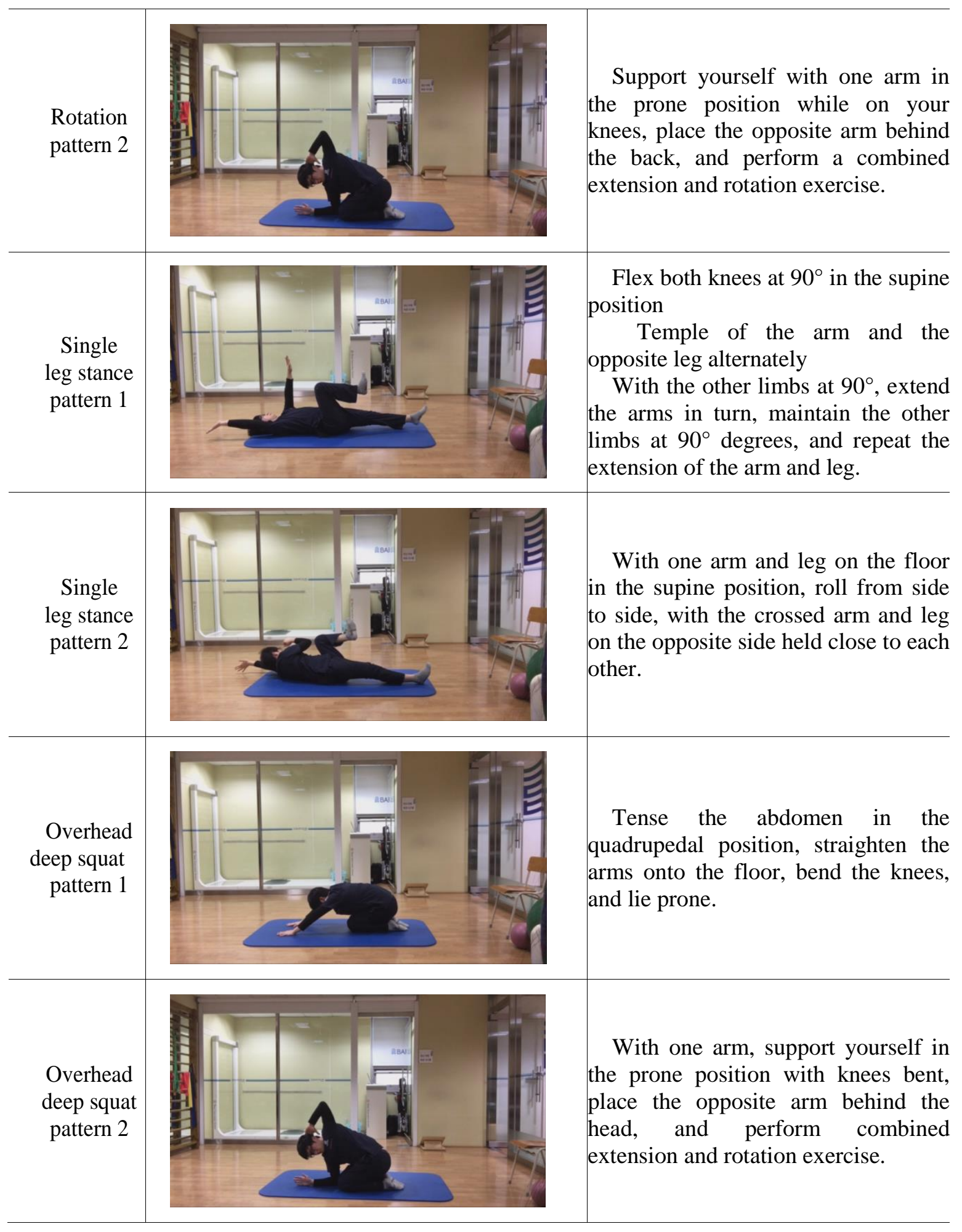

\subsection{Measurement}

2.3.1. Selective Functional Movement Assessment (Movement Screening): Selective functional movement assessment (SFMA) is used to evaluate normal functional movement patterns (Table 2). It is also used to evaluate dysfunctional movement with seven types of movement tests in patients with musculoskeletal disorders [28]. SFMA has also been performed in individuals without musculoskeletal disorders. As a result, high 
confidence values were obtained. For internal reliability of SFMA, the kappa coefficients between three evaluators were 0.72-0.83, indicating almost perfect agreement, and the agreement rate was $83-88 \%$ [31].

Table 2. SFMA Assessment

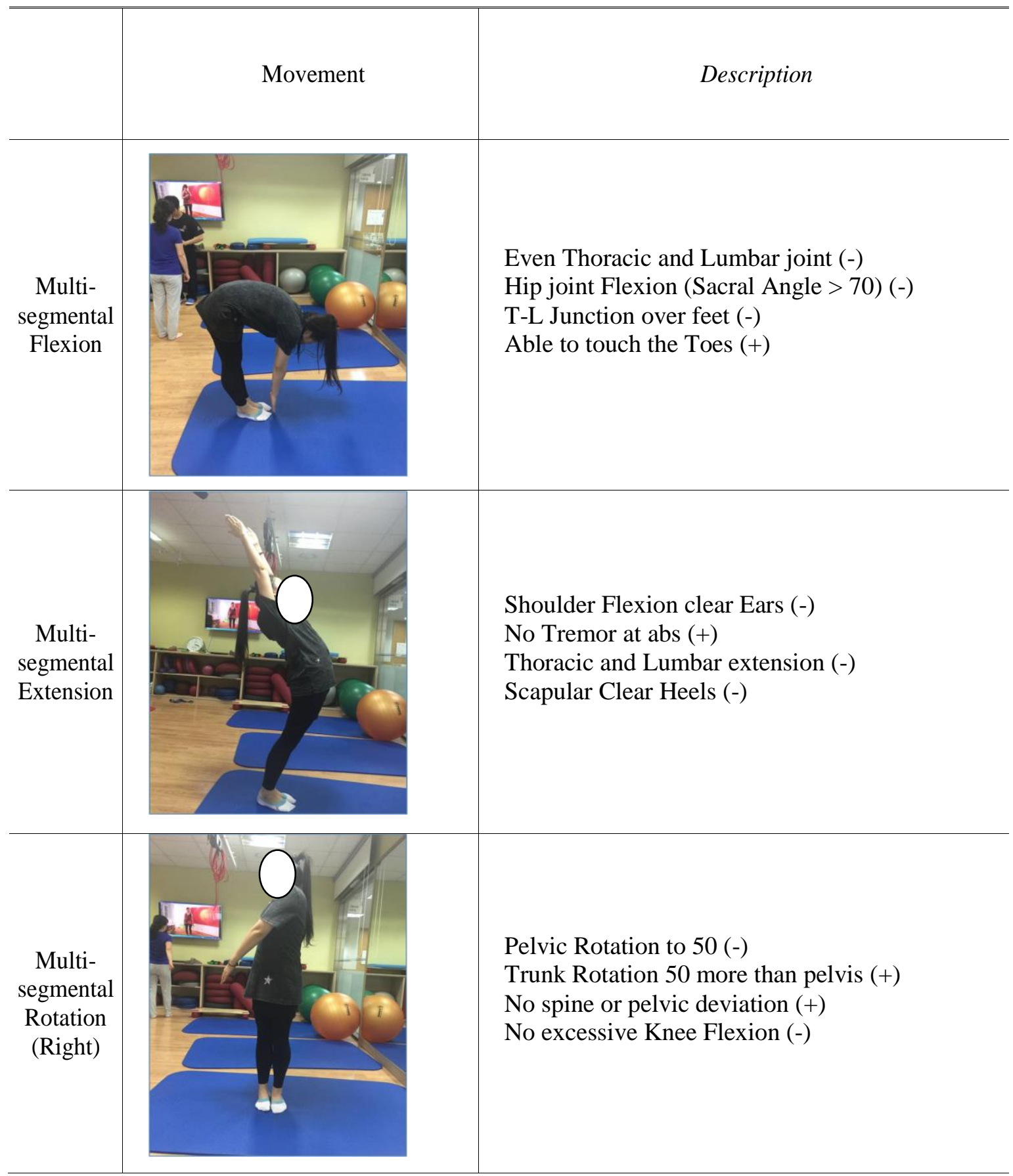




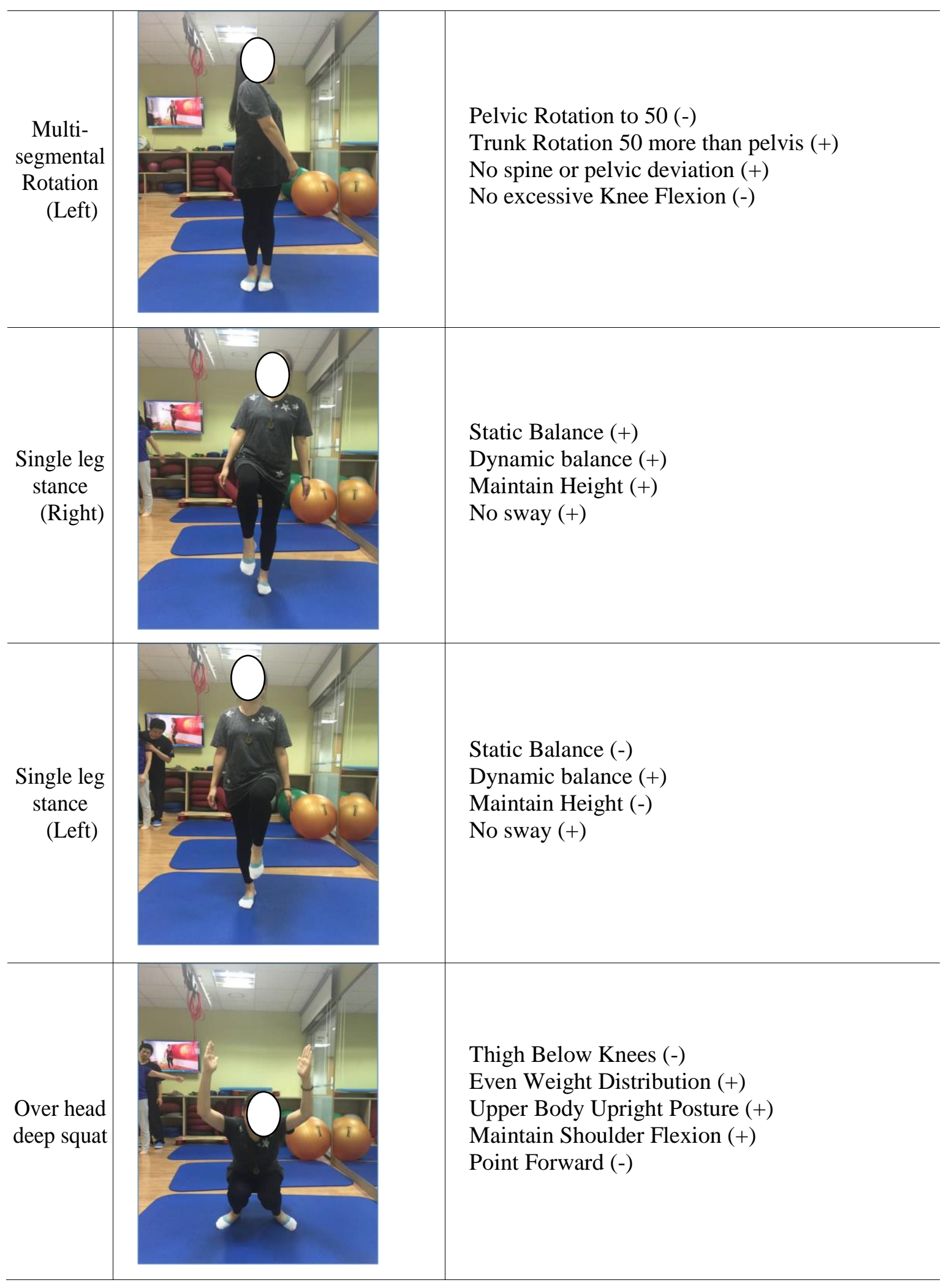

2.3.2. Straight Leg Raising Test (Movement Screening): The straight leg raising test is used to examine the flexibility of the lower extremity as part of FMS. According to the criteria of the Korean FMA version, 3 points are given if a bar is raised from the ankle to a level between the thigh and anterior superior iliac spine of the opposite lower extremity, 
2 points are given if the bar is raised to a level between the thigh and patella of the opposite lower extremity, 1 point is given if the bar is raised to a level lower than the patella, and 0 points are given if the subject has pain or the test is not properly performed [29]. The straight leg raising test is performed actively or passively [30]. Thus, if the test cannot be performed actively, it is attempted passively, based on structural or motor control problems.

2.3.3. Kujala Scoring Questionnaire: The Kujala score is not well known in Korea; it was developed by Dr. Urho Kujala in 1993, specifically for use with PPS. The score is based on a total of 13 categories: 1. Limp, 2. Support, 3. Walking, 4. Stairs, 5. Squatting, 6. Running, 7. Jumping, 8. Prolonged sitting with knee flexed, 9. Pain, 10. Swelling, 11. Abnormal painful kneecap movement, 12. Atrophy of thigh, and 13. Flexion deficiency. Associated questions vary according to items: function, pain, and structure; the measurement of each item is set according to the characteristics.

2.3.4. Visual Analogue Scale (VAS): The VAS is a commonly used and simple pain assessment tool [34]. In the present study, numbers were entered at $1-\mathrm{cm}$ intervals on a $10-\mathrm{cm}$ scale, with 10 considered most painful and 0 not at all painful.

\section{Results}

The results of the present study are as follows: in SFMA, Multi-Segmental Flexion (MSF) showed improvement from Dysfunctional Non-painful (DN) to Functional Nonpainful (FN); Multi-Segmental Extension (MSE) showed improvement from Dysfunctional painful (DP) to FN; Single Leg Stance (SLS) (both right and left) showed improvement from DN to FN; and Overhead Deep Squat (ODS) showed improvement from DP to FN (table 1). The Straight Leg Raising (SLR) test showed an increase on the right from $82^{\circ}$ to $90^{\circ}$ and from $63^{\circ}$ to $81^{\circ}$ on the left (table 2). The Kujala score for pain assessment and activities of daily living increased from 63 to 94 points (table 3 ). The VAS for pain improved from 8 to 3 (table 4).

Table 3. SFMA(Movement screening)

\begin{tabular}{lcc}
\hline & \multicolumn{1}{c}{ Pre-test } & Post-test \\
\hline MSF & Dysfunctional Non-painful & Functional Non-painful \\
MSE & Dysfunctional painful & Functional Non-painful \\
MSR (right) & Dysfunctional Non-painful & Dysfunctional Non-painful \\
MSR (left) & Dysfunctional Non-painful & Dysfunctional Non-painful \\
SLS (Right) & Dysfunctional Non-painful & Functional Non-painful \\
SLS (Left) & Dysfunctional Non-painful & Functional Non-painful \\
ODS & Dysfunctional painful & Functional Non-painful \\
\hline
\end{tabular}


Table 4. Straight Leg Raise Test (Movement Screening)

\begin{tabular}{lcc}
\hline & Pre-test & Post-test \\
\hline Right & $82^{\circ}$ & $90^{\circ}$ \\
Left & $63^{\circ}$ & $81^{\circ}$ \\
\hline
\end{tabular}

Table 5. Kujala Scoring Questionnaire

\begin{tabular}{lcc}
\hline & Pre-test & Post-test \\
\hline 1. Limp & 3 & 5 \\
2. Support & 3 & 5 \\
3. Walking & 3 & 5 \\
4. Stairs & 4 & 8 \\
5. Squatting & 3 & 5 \\
6. Running & 6 & 8 \\
7. Jumping & 2 & 10 \\
8. Prolonged sitting with knee flexed & 6 & 10 \\
9. Pain & 6 & 8 \\
10. Swelling & 10 & 10 \\
11. Abnormal painful kneecap movement & 10 & 10 \\
12. Atrophy of thigh & 3 & 5 \\
13. Flexion deficiency & 3 & 5 \\
& $63 / 100$ & $94 / 100$ \\
\hline
\end{tabular}

Table 6. Visual Analogue Scale

\begin{tabular}{lcc}
\hline & Pre-test & Post-test \\
\hline Score & 8 & 3 \\
\hline
\end{tabular}

\section{Discussion}

The present study was conducted to identify the effects of SFMA-based correction exercises on knee joint pain in a patient with PPS following pregnancy. Based on prior research on movement assessment, the SFMA and SLR, rather than the Clark test [35] or $\mathrm{Q}$ angle [36] used in the diagnosis of PPS, were adopted as an assessment tool. The Kujala scoring questionnaire and VAS were used to assess pain. The results were as follows.

In the SFMA screening, multi-joint extension improved from DN to FN, multi-joint rotation (right) was unchanged at $\mathrm{DN}$, multi-joint rotation (left) was unchanged at $\mathrm{DN}$, 
one-leg standing (right) improved from DN to FN, one-leg standing (left) improved from DN to FN, and the overhead deep squat changed from DP to DN. As suggested by Petersen et al. (2014), pain in multi-joint extension and overhead deep squat with manual therapy for correction and mobility indicates that PPS is not structural but instead functional or caused by dynamic imbalance.

The straight leg raising test increased on the right from $82^{\circ}$ to $90^{\circ}$ and from $63^{\circ}$ to $81^{\circ}$ on the left. The Kujala score increased from 63 to 94 . Given that the angular difference affected the degree of pain, it was verified again that shortening of the hamstring affects patellar pain, as reported by White, Dolphin, \& Dixon (2009). The VAS score improved from 8 to 3 . In conclusion, the findings suggest that PPS should be managed as a functional or operational imbalance, as reported in multiple prior studies [11-15]. Future research requires study of a larger number of subjects, and PPS mediated through a variety of motor controls should be studied.

\section{Acknowledgements}

This study was supported by Sahmyook University

\section{References}

[1] P. Baquie and P. Brukner, "Injuries presenting to an Australian sports medicine centre: a 12-month study", Clinical Journal of Sport Medicine, vol. 7, no. 1, (1997), pp. 28-31.

[2] J. E. Taunton, “A retrospective case-control analysis of 2002 running injuries”, British journal of sports medicine, vol. 36, no. 2, (2002), pp. 95-101.

[3] D. T. Reilly and M. Martens, "Experimental analysis of the quadriceps muscle force and patello-femoral joint reaction force for various activities", Acta Orthopaedica Scandinavica, vol. 43, no. 2, (1972), pp. 126-137.

[4] S. Dixit, "Management of patellofemoral pain syndrome", Am Fam Physician, vol. 75, no. 2, (2007), pp. 194-202.

[5] S. R. Piva, E.A. Goodnite and J. D. Childs, "Strength around the hip and flexibility of soft tissues in individuals with and without patellofemoral pain syndrome", Journal of orthopaedic \& sports physical therapy, vol. 35, no. 12, (2005), pp. 793-801.

[6] L. C. White, P. Dolphin, and J. Dixon, "Hamstring length in patellofemoral pain syndrome", Physiotherapy, vol. 95, no. 1, (2009), pp. 24-28.

[7] J. H. Lee and W. H. Lee, "Biochemical comparison of patellofemoral pain syndromes in elite athlets with lower extrimoty in normal athletesm", Magazine of Coaching Capabilities Development, vol. 12, no. 4, (2010), pp. 119-126.

[8] K. Kiesel, P. J. Plisky and M. L. Voight, "Can serious injury in professional football be predicted by a preseason functional movement screen”, N Am J Sports Phys Ther, vol. 2, no. 3, (2007), pp. 147-158.

[9] G. Cook, "Movement: Functional movement systems: Screening, assessment, corrective strategies", On Target Publications, (2010).

[10] S. A. Choi, "Effects of ankle dorsiflexion on vastus medialis oblique and vastus lateralis muscle activity during straight leg raise exercise with hip external rotation in patellofemoral pain syndrome", Journal of Musculoskeletal Pain, vol. 22, no. 3, (2014), pp. 260-267.

[11] K. M. Crossley, "Performance on the single-leg squat task indicates hip abductor muscle function", The American journal of sports medicine, vol. 39, no. 4, (2011), pp. 866-873.

[12] W. Petersen, "Patellofemoral pain syndrome", Knee Surgery, Sports Traumatology, Arthroscopy, vol. 22, no. 10 , (2014), pp. 2264-2274.

[13] H. Negahban, "The effects of muscle fatigue on dynamic standing balance in people with and without patellofemoral pain syndrome", Gait \& posture, vol. 37, no. 3, (2013), pp. 336-339.

[14] P. Lucareli, "Dynamic knee valgus" mechanism during the landing phase of a triple hop test in women with patellofemoral pain syndrome: A comparison to painfree controls", Gait \& posture, vol. 39, (2014), pp. S129-S130.

[15] J. Foley, "Findings of the Lower Extremity Dynamic Screen in Patients with Patellofemoral Pain Syndrome: A Pilot Study", (2013).

[16] T. Graven-Nielsen, "Inhibition of Maximal Voluntary Contraction Force by Experimental Muscle Pain: A Centrally Mediated Mechanism”, Muscle \& nerve, vol. 26, no. 5, (2002), pp. 708-712.

[17] P. W. Hodges and G.L. Moseley, "Pain and motor control of the lumbopelvic region: effect and possible mechanisms", Journal of Electromyography and Kinesiology, vol. 13, no. 4, (2003), pp. 361-370.

[18] M. K. Sohn, "Inhibition of Motor Unit Firing during Experimental Muscle Pain in Humans", Muscle \& nerve, vol. 23, no. 8, (2000), pp. 1219-1226. 
[19] L. Arendt-Nielsen and T. Graven-Nielsen, "Muscle Pain: Sensory Implications and Interaction With Motor Control", The Clinical journal of pain, vol. 24, no. 4, (2008), pp. 291-298.

[20] U. F. Ervilha, "Experimental muscle pain changes motor control strategies in dynamic contractions", Experimental brain research, vol. 164, no. 2, (2005), pp. 215-224.

[21] P. W. Hodges, "Experimental muscle pain changes feedforward postural responses of the trunk muscles", Experimental brain research, vol. 151, no. 2, (2003), pp. 262-271.

[22] G. L. Moseley and P. W. Hodges, "Reduced Variability of Postural Strategy Prevents Normalization of Motor Changes Induced by Back Pain: A Risk Factor for Chronic Trouble?”, Behavioral neuroscience, vol. 120, no. 2, (2006), pp. 474-476.

[23] E. Garvin, "Pain in pregnancy", Case Studies in Pain Management, (2014), pp. 282.

[24] D. R. Karnad and K. K. Guntupalli, "Neurologic disorders in pregnancy", Critical care medicine, vol. 33, no. 10, (2005), pp. S362-S371.

[25] G. Spahn, "[Knee-related Pain Problems during Pregnancy Correlate with an Increase in Body Weight. Results of a Prospective Study]", Zeitschrift fur Geburtshilfe und Neonatologie, vol. 219, no. 5, (2015), pp. 213-220.

[26] S. Brown and J. Lumley, "Maternal health after childbirth: results of an Australian population based survey”, BJOG: An International Journal of Obstetrics \& Gynaecology, vol. 105, no. 2, (1998), pp. 156161.

[27] A. J. T. Connolly and L. Pahel, "Effects of pregnancy and childbirth on postpartum sexual function: a longitudinal prospective study", International Urogynecology Journal, vol. 16, no. 4, (2005), pp. 263267.

[28] J. Izraelski, "Movement: Functional Movement Systems: Screening, Assessment, and Corrective Strategies", The Journal of the Canadian Chiropractic Association, vol. 56, no. 2, (2012), pp. 158.

[29] S. H. Ahn, and J. H. Lee, Confidence and validity in the Korean version of the functional movement screen, the Koean Society of Physical Theraphy, (2010).

[30] G. Cook, "Movement: Functional movement systems", Screening-Assessment-Corrective Strategies. Chichester (UK), Lotuspublishing, (2010). 